\title{
Early experience with laparoscopic treatment of liver tumors using a separable cluster electrode with a no-touch technique
}

\author{
Hyun Pyo Hong ${ }^{1}$, Jee Youn Lee ${ }^{2}$, Mi Yeon Lee³, Kyung Uk Jung², Byung Ik Kim, Byung Ho Son², Jun Ho Shin², \\ Sung Ryol Lee ${ }^{2}$ \\ 1Department of Radiology, Kangbuk Samsung Hospital, Sungkyunkwan School of Medicine, University, Seoul, Republic of Korea \\ ${ }^{2}$ Department of Surgery, Kangbuk Samsung Hospital, Sungkyunkwan School of Medicine, University, Seoul, Republic of Korea \\ ${ }^{3}$ Division of Biostatistics, Department of R\&D Management, Kangbuk Samsung Hospital, Sungkyunkwan School of Medicine, University, \\ Seoul, Republic of Korea \\ ${ }^{4}$ Division of Gastroenterology, Department of Internal Medicine, Kangbuk Samsung Hospital, Sungkyunkwan School of Medicine, \\ University, Seoul, Republic of Korea
}

Videosurgery Miniinv DOI: https://doi.org/10.5114/wiitm.2020.95065

\begin{abstract}
Introduction: Radiofrequency ablation (RFA) is one of the best curative treatments for hepatocellular carcinoma in selected patients, and this procedure can be applied either percutaneously or laparoscopically. Laparoscopic RFA has the benefit of direct visual control of the RFA procedure. Cluster electrodes (Octopus RF electrodes) can create a common ablation zone.

Aim: Using these two methods (laparoscopic approach and no touch technique), this present study evaluated the technical and clinical outcomes of early experience with laparoscopic RFA and a no-touch technique.

Material and methods: Between November 2015 and November 2018, 21 patients underwent laparoscopic RFA for hepatocellular carcinoma with a no-touch technique using cluster electrodes. Laparoscopic RFA is recommended for patients with a contraindication for surgical resection, patients wants and a relative contraindication for conventional percutaneous RFA, such as lesions adjacent to the gastrointestinal tract, gallbladder, bile duct, or heart.

Results: In the 21 tumors, 2 were treated with a single electrode, 12 with 2 electrodes, and 7 tumors with 3 electrodes. The mean time of ablation per lesion was $20.43 \pm 8.77 \mathrm{~min}$. There was no mortality, local tumor progression, delayed destructive biliary damage, or liver abscess at the follow-up computed tomography. No technical failures occurred. Conclusions: Laparoscopic RFA can access lesions for which percutaneous RFA is contraindicated or risky. Cluster electrodes can create sufficient ablation zones without contact and can achieve a sufficient margin with a low complication rate and no tumor dissemination. Therefore, laparoscopic RFA with a no-touch technique might be a safe and feasible treatment for HCC tumor in selected patients.
\end{abstract}

Key words: hepatocellular carcinoma, radiofrequency ablation, laparoscopic radiofrequency ablation, cluster electrodes.

\section{Introduction}

Hepatocellular carcinoma (HCC) is one of the most frequent primary hepatic malignancies [1-4]. Liver transplantation is an optimal therapy for many early diagnosed HCC, but it is limited by the availability of organs. Resection is often contraindicated due to limited liver function. Hence, several alternative non-surgical treatments to potentially cure HCC

\section{Address for correspondence}

Sung Ryol Lee MD, PhD, Department of Surgery, Kangbuk Samsung Hospital, Sungkyunkwan School of Medicine, University, 29 Saemunan-ro, Jongno-gu, Seoul 03181, Republic of Korea, phone: +82 220018404,821063382722 , fax: +82 2 2001 2131, e-mail: sungryol.lee@samsung.com 
have been developed. Since the early 1990s, there have been reported results about thermal ablation by radiofrequency (RF) in several alternative treatments [5-7]. In addition, because of many technical advances, radiofrequency ablation (RFA) can be applied percutaneously, laparoscopically, or during open surgery.

In 1997, Curley et al. performed a feasibility study of laparoscopic RFA on pigs [8], and favorable results were subsequently achieved in preliminary clinical experience [9-11]. In several studies, the laparoscopic approach yielded a significantly lower rate of local recurrence compared to the percutaneous approach [12, 13]. The laparoscopic approach has the benefits of direct visual control of the RFA procedure, exposure and isolation of the liver from the surrounding tissue, and effective management of intraoperative bleeding [14].

Many hospitals continue to use conventional RFA devices, which employ a single-needle electrode that delivers an electrical current at $400-500 \mathrm{kHz}$, to create a core zone of ablation at the tumor site that extends radially into the adjacent tissue. The single-needle tip electrode can reach target temperatures of over $100^{\circ} \mathrm{C}$, but is prone to rapid charring, thereby increasing tissue impedance, which limits thermal energy distribution.

In this study, RF procedures were performed using separable cluster RF electrodes (Octopus, STARmed; Goyang-si, Gyunggi-do, Republic of Korea), a multi-channel switching radiofrequency system (STARmed), and an RF generator. Because the separable cluster electrode consists of one adapter and three active applicators which can be separated into three independent applicators, it can create a sufficient large ablative volume including the tumor and a safety margin with no-touch technique.

\section{Aim}

Through these two methods, this study evaluated the technical and clinical outcomes of early experience of laparoscopic RFA with no-touch technique. To our knowledge, this study is the first attempt to assess early experience with laparoscopic RFA and a no-touch technique with switching monopolar RFA using a separable cluster electrode.

\section{Material and methods}

Between November 2015 and November 2018, 21 patients underwent laparoscopic RFA for HCC with a no-touch technique. All patients reported their personal hospital history and underwent physical examination, retrospectively; screening for hepatitis B and hepatitis C; and serum laboratory tests assessing liver function, hemostasis, renal function, and determination of serum $\alpha$-fetoprotein level (AFP). Abdominal computed tomography (CT) and magnetic resonance imaging (MRI) were used to assess local tumor extension.

The indications for laparoscopic RFA are similar to those for conventional percutaneous RFA: three or fewer tumors measuring $<3 \mathrm{~cm}$ in diameter or a solitary tumor with a major axis $<5 \mathrm{~cm}$. Laparoscopic RFA is highly recommended for patients with a contraindication for surgical resection, patients wants and a relative contraindication for conventional percutaneous RFA, such as lesions adjacent to the gastrointestinal tract, gallbladder, bile duct, or heart [15]. Contraindications specific to laparoscopic RFA are the same as those for generic laparoscopy and include previous abdominal surgery, cardiopulmonary disorders, and severe obesity.

\section{Laparoscopic RFA procedure}

All patients were placed in a supine position after induction of general anesthesia. After pneumoperitoneum $\left(\mathrm{CO}_{2}\right.$ at $\left.12 \mathrm{~mm} \mathrm{Hg}\right)$ was achieved, exploration was performed with a flexible laparoscope through a $12 \mathrm{~mm}$ umbilical port. Another $10 \mathrm{~mm}$ port was created at the midline or the subxiphoid in the abdomen. One additional $5 \mathrm{~mm}$ right or left lateral subcostal port was placed, depending on the location of the tumor. The liver was laparoscopically fully mobilized from the retroperitoneum and diaphragm to access the tumor-bearing segments. In the case of the gallbladder, if the tumor was attached to the gallbladder, RFA was performed after cholecystectomy. For intestinal adhesions, RFA was performed safely by separating it from liver tissue through laparoscopic dissection. The ability to drive the liver in various directions is also important to provide broad access for electrode placement. Through US guidance, the electrode were inserted at the periphery of the tumor with laparoscopic visualization. During the operation, the location of the tumor was confirmed through laparoscopic US, and it was possible to apply it to the tumor inside the parenchyma of the liver. The RFA procedure was monitored by intraoperative US. The separa- 
ble cluster electrode and a multi-channel switching radiofrequency system (STARmed) were used. The Octopus electrode is composed of three, cooled tip electrodes with a $2 \mathrm{~cm}, 2.5 \mathrm{~cm}$, and $3 \mathrm{~cm}$ active tip which has a $50-\mathrm{cm}$-long, flexible cable. A special adapter connects the three cable to one piece. The distance of each electrode was determined by the operator based on the size of the tumor. After placing the separable electrode, the radiofrequency energy was delivered for approximately 8-12 $\mathrm{min}$ until the color of the tumor and adjacent hepatic tissue turned to yellow. If the ablative margin was not sufficient, radiofrequency energy was additionally delivered after repositioning the electrodes to a different site. A 200-watt, multichannel, radiofrequency system with three independently adjustable generators allowed independent control of radiofrequency energy delivery to each electrode. For tissue heating, the radiofrequency energy was applied to two of the three electrodes; the radiofrequency energy was delivered in an alternating fashion to the three possible pairs of electrodes of the separable electrode. The switching of energy delivery between the pairs of electrodes was automatically controlled by the multi-channel switching radiofrequency system according to the continuously measured impedance values. The algorithm of energy delivery was based on the manufacturer's instructions. During ablation, continuous irrigation of the needle with cold saline was provided with an external pump. Because the Octopus RF electrodes can produce ablation zones of $4.5 \mathrm{~cm}$ with a single placement of electrodes, 1,2 or 3 electrodes around the tumor can create a common ablation zone. Using this method, we could ablate tumors without contact (Photo 1).

\section{Postoperative imaging and follow-up}

The follow-up study after RFA was conducted according to our hospital regimen. Ultrasound (contrast enhanced, if needed) examination, computed tomography, and measurement of $\alpha$-fetoprotein level were performed every 3 months. Magnetic resonance imaging (MRI) was performed when needed.

\section{Ethics}

The study was approved by the Ethical Review Board of the general surgery committee at the hospital.

\section{Statistical analysis}

Statistical analysis was performed using STATA version 14.0 (Stata Corp LP, College Station, TX, USA).

\section{Results}

The characteristics of patients undergoing RFA are shown in Table I. From November 2015 to November 2018, 21 patients were treated with laparoscopic RFA with a no-touch technique. Of the 21 patients, 14 were male and 7 female. The mean diameter of the $21 \mathrm{HCCS}$ was $20.43 \pm 8.77 \mathrm{~mm}$.

Tumor characteristics and the results of RFA are shown Table II. There was no technical failure. In the 21 tumors, 2 were treated with a single electrode, 12 with 2 electrodes, and 7 tumors with 3 electrodes. The mean time of ablation per lesion was 16.52 $\pm 6.29 \mathrm{~min}$. Seventeen tumors were located in the liver dome, 2 tumors were located in the right anterior portion, 1 tumor was located in the right posterior portion and 1 tumor was located in the caudate lobe.

There were 2 complications (ascites and pleural effusion) related to ablation. According to the Dindo-Clavien classification, the complication was minor (grade I). No patients developed perioperative neoplastic seeding, liver abscess, gastrointestinal perforation, gastrointestinal bleeding, or inferior vena cava thrombosis. Three or 6 months later, there was no mortality, local tumor progression, delayed destructive biliary damage, or liver abscess at the follow-up CT.

\section{Discussion}

Liver transplantation or resection is the optimal therapy for early diagnosed HCC, but there are several limitations. Therefore, several alternative non-surgical treatments to potentially cure HCC have been developed. Over several decades, RFA has become a good treatment method for HCC with resection. Shiina et al. [16] reported that RFA could be a locally feasible and safe procedure based on the 10-year outcomes of RFA in patients with HCC.

Conventional RFA is a relatively easy procedure and does not require surgery. However, because of the potential for thermal injury, a tumor adjacent to organs is not readily accessible. During the RFA procedure, the incidence of organ injury varies from $0.5 \%$ to $0.7 \%$ in the literature [17]. Although the incidence of organ injury is not frequent, most compli- 

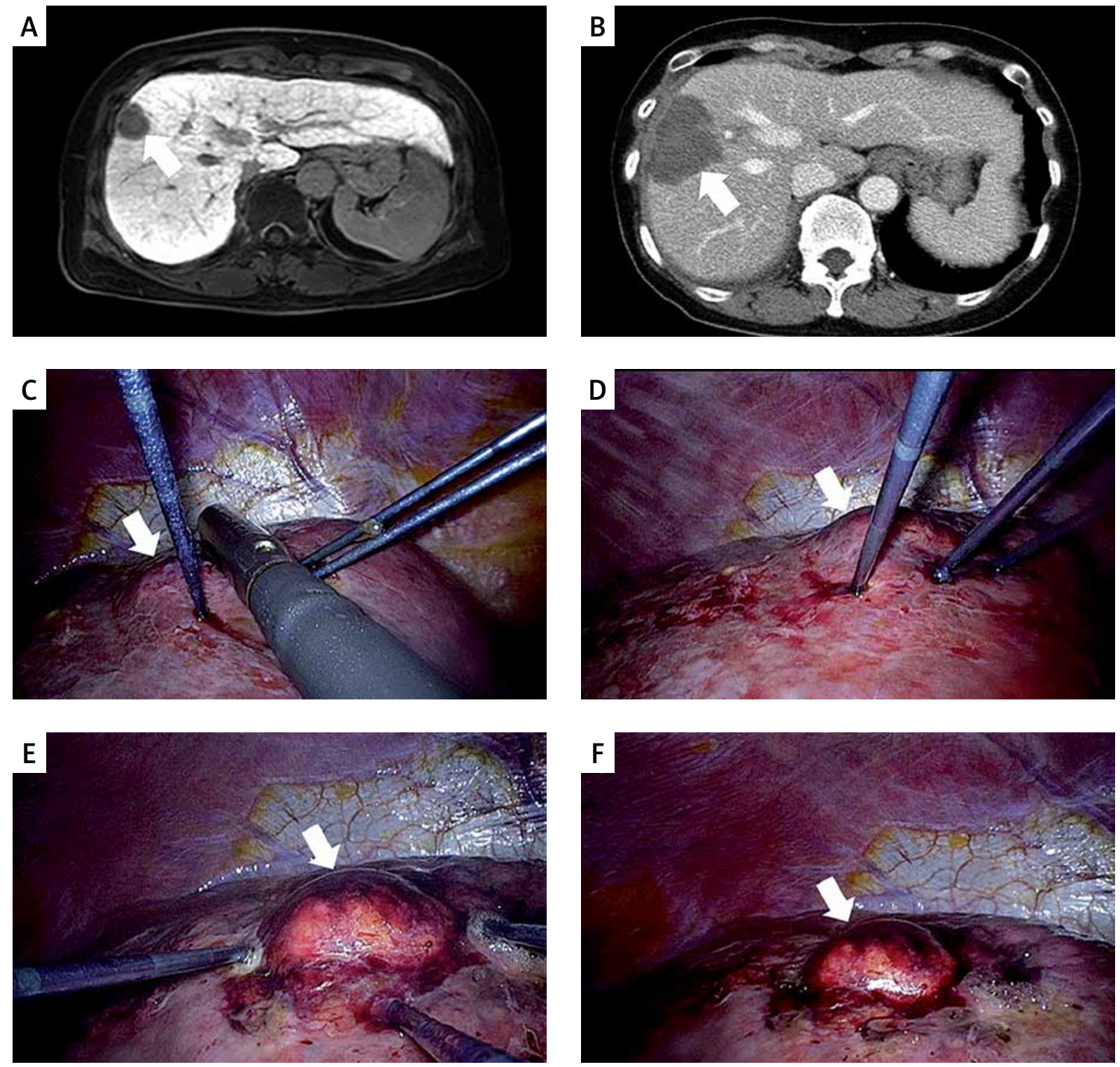

Photo 1. Laparoscopic radiofrequency ablation for hepatocellular carcinoma. A - Pre-therapeutic CT: $30 \mathrm{~mm}$ $\mathrm{HCC}$ at S4/8, B - post-therapeutic CT, C - $1^{\text {st }}$ RFA, D - $2^{\text {nd }}$ RFA, E - $3^{\text {rd }}$ RFA, F - final features after RFA

cations are fatal even if the patients are diagnosed early and adequately treated. Additionally, conventional RFA directly contacts the tumor. Therefore, during RFA or electrode removal, tumor seeding can occur, especially with surface lesions.

The advantages of the laparoscopic approach include the ability to see and control the entire procedure, to separate the liver from the surrounding tissue, and to control intraoperative complications [14]. RFA of an HCC on the superior or inferior surface of the liver can relatively easily result in injury of the adjacent abdominal wall or adjacent organ, with the possibility of major complications and tumor seeding [18-20]. Therefore, the laparoscopic approach is frequently recommended in superficial or extrahepatic protrusive HCC and HCC adjacent to a major organ including the heart $[21,22]$. In this study, 17 tumors were located in the liver dome, 2 tumors were located in the right anterior portion, 1 tumor was located in the right posterior portion and 1 tumor was located in the caudate lobe. Most of the tumors were expected to be difficult to access with conventional RFA. However, during laparoscopic RFA, we observed the whole procedure, and no organ injury occurred. 
Table I. Baseline characteristics of patients undergoing RF ablation

\begin{tabular}{|lc|}
\hline Variable & Value \\
\hline Age, mean \pm SD & $59.6 \pm 7.2$ \\
\hline Gender (M/F) & $14 / 7$ \\
\hline Liver cirrhosis: & 16 \\
\hline HBV & 1 \\
\hline HCV & 0 \\
\hline Alcoholic & 4 \\
\hline None & $4.08 \pm 0.67$ \\
\hline Albumin, mean \pm SD [g/dl] & $12.90 \pm 1.80$ \\
\hline Prothrombin time, mean \pm SD [s] & $1.10 \pm 0.62$ \\
\hline Total bilirubin, mean \pm SD [mg/dl] & $130.05 \mathrm{k} \pm 75.10$ \\
\hline Platelets, mean \pm SD $\left[\times 10^{3} / \mathrm{mm}^{3}\right]$ & $32.62 \pm 14.51$ \\
\hline AST, mean \pm SD [IU/l] & $26.10 \pm 16.69$ \\
\hline ALT, mean \pm SD [IU/l] & $19: 2: 0$ \\
\hline Child Pugh class (A : B : C) & \\
\hline
\end{tabular}

ALT - alanine transaminase, $A S T$ - aspartate amino transferase, $H B V$ - hepatitis $B$ virus, $H C V$ - hepatitis $C$ virus, $R F$ - radiofrequency.

With laparoscopic RFA, simultaneous procedures such as a cholecystectomy and unroofing of a hepatic cyst can be performed. In this study, 2 patients underwent simultaneous cholecystectomy. During laparoscopic RFA, intraoperative US is used routinely and can increase correct RF electrode placement, achieve a sufficient margin and evaluate the efficacy of ablation [23-25]. Additionally, the laparoscopic approach using pneumoperitoneum has an advantage over the conventional RFA because portal blood flow is reduced by about $40 \%$, allowing for better thermal conduction, which contributes to better ablation efficacy [26].

With all of the above-mentioned advantages, no specific complication was observed in this study. Safety is one of the most important issues in minimally invasive therapies such as laparoscopic RFA. Complications after laparoscopic RFA include liver failure, bile duct thermal injury, liver abscess, trocar injury, postoperative bleeding from the mesentery, and gastrointestinal bleeding. Complications specific to laparoscopic RFA are pneumonia, pneumothorax, trocar injury, and postoperative bleeding from the mesentery or abdominal wall. In this study of HCC patients who underwent laparoscopic RFA, only

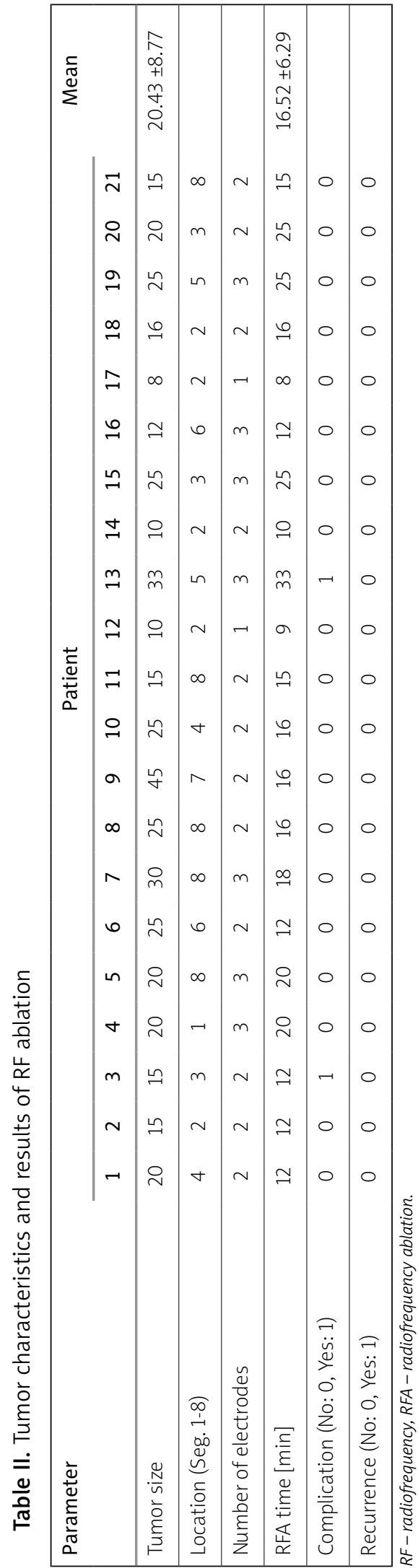


2 patients had a complication (ascites and pleural effusion) after RFA. According to the Dindo-Clavien classification, the complication was minor (grade I) (Table II) and did not require any pharmacological treatment or surgical and radiological interventions.

Conventional laparoscopic RFA was performed by direct application to the tumor. Therefore, during the RFA procedure or after removal of the electrode after RFA, tumor dissemination can occur, especially with superficial tumors. The local recurrence rate of laparoscopic RFA varies from 0 to $12 \%$ [27-34]. The risk of local recurrence increases with an increase in HCC size, but the local recurrence rate differs markedly if a circumferential 5-mm safety margin can be secured. In this study, the RFA cluster electrodes can produce ablation zones of $4.5 \mathrm{~cm}$ with a single electrode, and 2 or 3 switching electrodes around the tumor can create a common ablation zone (Photo 1 ). Using this cluster electrode, the RF needle can be inserted through normal liver tissue to avoid direct puncture and dissemination of the tumor. Additionally, sufficient safety margins were obtained by the broad ablation zone, and no marginal recurrence was noted in 21 tumors during the follow-up period.

This study had major limitations, including its retrospective nature, the absence of a control group, the short follow-up period, and a small number of patients. More long-term outcomes and a randomized control trial are necessary. However, this study was an initial experience with laparoscopic RFA and the no-touch technique.

\section{Conclusions}

The laparoscopic approach without tumor contact is more advanced than conventional laparoscopic RFA. This technique could be an approach for HCC that is not easily accessible by conventional percutaneous RFA. Additionally, the laparoscopic approach can be performed safely with a low rate of complications, without tumor dissemination during the procedure, and with a low marginal recurrence rate due to a sufficient margin with a broad ablation zone. In conclusion, laparoscopic RFA with the no-touch technique might be acceptable as a feasible and reliable therapy for HCC tumor in selected patients.

\section{Acknowledgments}

Hyun Pyo Hong and Jee Youn Lee are co-first authors and contributed equally to this work.

\section{Conflict of interest}

The authors declare no conflict of interest.

\section{References}

1. El-Serag HB, Mason AC. Rising incidence of hepatocellular carcinoma in the United States. N Engl I Med 1999; 340: 745-50.

2. Taylor-Robinson SD, Foster GR, Arora S, et al. Increase in primary liver cancer in the UK, 1979-94. Lancet 1997; 350: 1142-3.

3. Llovet JM, Burroughs A, Bruix J. Hepatocellular carcinoma. Lancet 2003; 362: 1907-17.

4. Okuda K, Okuda H. Primary liver cell carcinoma. In: Oxford Textbook of Clinical Hepatology, Mcintyre N, Benhamou JP, Bircher J, et al. (eds). Oxford University Press, Oxford 1991; 1019-52.

5. Poon RT, Fan ST, Tsang FH, Wong J. Locoregional therapies for hepatocellular carcinoma: a critical review from the surgeon's perspective. Ann Surg 2002; 235: 466-86.

6. Lu MD, Xu HX, Xie XY, et al. Percutaneous microwave and radiofrequency ablation for hepatocellular carcinoma: a retrospective comparative study. J Gastroenterol 2005; 40: 1054-60.

7. Seki S, Sakaguchi H, Kadoya H, et al. Laparoscopic microwave coagulation therapy for hepatocellular carcinoma. Endoscopy 2000; 32: 591-7.

8. Curley SA, Davidson BS, Fleming RY, et al. Laparoscopically guided bipolar radiofrequency ablation of areas of porcine liver. Surg Endosc 1997; 11: 729-33.

9. Cuschieri A, Bracken J, Boni L. Initial experience with laparoscopic ultrasound-guided radiofrequency thermal ablation of hepatic tumours. Endoscopy 1999; 31: 318-21.

10. Siperstein A, Garland A, Engle K, et al. Laparoscopic radiofrequency ablation of primary and metastatic liver tumors. Technical considerations. Surg Endosc 2000; 14: 400-5.

11. Goletti O, Lencioni R, Armillotta N, et al. Laparoscopic radiofrequency thermal ablation of hepatocarcinoma: preliminary experience. Surg Laparosc Endosc Percutan Tech 2000; 10: 284-90.

12. Mulier S, Ni Y, Jamart J, et al. Local recurrence after hepatic radiofrequency coagulation: multivariate meta-analysis and review of contributing factors. Ann Surg 2005; 242: 158-71.

13. Mulier S, Ruers T, Jamart J, et al. Radiofrequency ablation versus resection for resectable colorectal liver metastases: time for a randomized trial? An update. Dig Surg 2008; 25: 445-60.

14. Topal B, Aerts R, Penninckx F. Laparoscopic radiofrequency ablation of unresectable liver malignancies: feasibility and clinical outcome. Surg Laparosc Endosc Percutan Tech 2003; 13: 11-5.

15. Kudo M. Local ablation therapy for hepatocellular carcinoma: current status and future perspectives. I Gastroenterol 2004; 39: 205-14.

16. Shiina S, Tateishi R, Arano T, et al. Radiofrequency ablation for hepatocellular carcinoma: 10-year outcome and prognostic factors. Am J Gastroenterol 2012; 107: 569-77; quiz 578.

17. Fonseca AZ, Santin S, Gomes LG, et al. Complications of radiofrequency ablation of hepatic tumors: frequency and risk factors. World J Hepatol 2014; 6: 107-13.

18. Stigliano R, Marelli L, Yu D, et al. Seeding following percutaneous diagnostic and therapeutic approaches for hepatocellular carcinoma. What is the risk and the outcome? Seeding risk for percutaneous approach of HCC. Cancer Treat Rev 2007; 33: 437-47. 
19. Livraghi T, Solbiati L, Meloni MF, et al. Treatment of focal liver tumors with percutaneous radio-frequency ablation: complications encountered in a multicenter study. Radiology 2003; 226: 441-51.

20. Llovet JM, Vilana R, Bru C, et al. Increased risk of tumor seeding after percutaneous radiofrequency ablation for single hepatocellular carcinoma. Hepatology 2001; 33: 1124-9.

21. Yokoyama T, Egami K, Miyamoto M, et al. Percutaneous and laparoscopic approaches of radiofrequency ablation treatment for liver cancer. J Hepatobiliary Pancreat Surg 2003; 10: 425-7.

22. Noguchi O, Izumi N, Kawamura H, et al. Radiofrequency tumor ablation for hepatocellular carcinoma therapeutic significance of approaching methods and the device differences. Jpn J Hyperthermia Oncol 2002; 18: 21-30.

23. Cillo $U$, Vitale A, Dupuis D, et al. Laparoscopic ablation of hepatocellular carcinoma in cirrhotic patients unsuitable for liver resection or percutaneous treatment: a cohort study. PLoS One 2013; 8: e57249.

24. Herbold T, Wahba R, Bangard C, et al. The laparoscopic approach for radiofrequency ablation of hepatocellular carcinoma: indication, technique and results. Langenbecks Arch Surg 2013; 398: 47-53.

25. de la Serna S, Vilana R, Sanchez-Cabus S, et al. Results of laparoscopic radiofrequency ablation for HCC. Could the location of the tumour influence a complete response to treatment? A single European centre experience. HPB (Oxford) 2015; 17: 387-93.

26. Odeberg S, Ljungqvist $O$, Svenberg T, et al. Haemodynamic effects of pneumoperitoneum and the influence of posture during anaesthesia for laparoscopic surgery. Acta Anaesthesiol Scand 1994; 38: 276-83.

27. Noguchi O, Izumi N, Inoue K, et al. Laparoscopic ablation therapy for hepatocellular carcinoma. Clinical significance of a newly developed laparoscopic sector ultrasonic probe. Dig Endosc 2003; 15: 179-84.

28. Chung MH, Wood TF, Tsioulias GJ, et al. Laparoscopic radiofrequency ablation of unresectable hepatic malignancies. A phase 2 trial. Surg Endosc 2001; 15: 1020-6.

29. Inamori H, Ido K, Isoda N, et al. Laparoscopic radiofrequency ablation of hepatocellular carcinoma in the caudate lobe by using a new laparoscopic US probe with a forward-viewing convex-array transducer. Gastrointest Endosc 2004; 60: 628-31.

30. Hildebrand P, Kleemann M, Roblick U, et al. Laparoscopic radiofrequency ablation of unresectable hepatic malignancies: indication, limitation and results. Hepatogastroenterology 2007; 54: 2069-72.

31. Santambrogio R, Podda M, Zuin M, et al. Safety and efficacy of laparoscopic radiofrequency ablation of hepatocellular carcinoma in patients with liver cirrhosis. Surg Endosc 2003; 17: 1826-32.

32. Santambrogio R, Opocher E, Costa M, et al. Survival and intra-hepatic recurrences after laparoscopic radiofrequency of hepatocellular carcinoma in patients with liver cirrhosis. J Surg Oncol 2005; 89: 218-25.

33. Machi J, Uchida S, Sumida K, et al. Ultrasound-guided radiofrequency thermal ablation of liver tumors: percutaneous, laparoscopic, and open surgical approaches. J Gastrointest Surg 2001; 5: 477-89.
34. Berber E, Siperstein A. Local recurrence after laparoscopic radiofrequency ablation of liver tumors: an analysis of $1032 \mathrm{tu}$ mors. Ann Surg Oncol 2008; 15: 2757-64.

Received: 21.02.2020, accepted: 21.04.2020. 